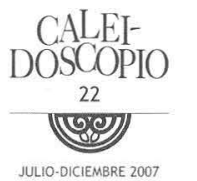

\title{
Procesos de socialización formativa del personal académico en México
}

LAURA ELENA PADILLA

$U A A$

\section{INTRODUCCIÓN}

L a educación superior está siendo sujeta cada vez con mayor intensidad a procesos de rendición de cuentas basados en la valoración de su calidad. Uno de los actores centrales en este contexto es el profesorado, ya que sobre éste recae de manera importante el desarrollo de las funciones clásicas de las Instituciones de Educación Superior (IES), esto es, de la docencia, la investigación y la difusión. Las características de la actividad del personal académico definen el nivel de consolidación de la profesión académica; de este modo, en la medida en que esta profesión se fortalezca se espera entonces que la calidad de sus funciones inherentes mejore.

Diversos estudios sobre la profesión académica resaltan la gran diversidad de sus miembros' ${ }^{1}$ que se genera en principio como resultado

P. G. Altbach, The International Academic Profession: Portraits of Fourteen Countries, Princeton, N. J., Carnegie Foundation for the Advancement of Teaching, 1996; M. Gil Antón et al., Los rasgos de la diversidad; Un estudio sobre los académicos mexicanos, Mèxico, Universidad Autónoma Metropolitana- Unidad Atzcapotzalco, 1994; R. Grediaga, Profesión académica, disciplinas y organizaciones, México, ANUIES, 2000. 
de sus dos principales ejes de diferenciación: el tipo de institución de educación superior y el de tipo de área disciplinar en el que ellos se formaron y en el que actualmente se desempeñan². Además, y para el caso de México, convendría agregar un tercer eje relativo al ejercicio profesional fuera del ámbito académico ${ }^{3}$. De esta manera, es importante que los estudios que se lleven a cabo en este ámbito distingan las diferencias en la socialización académica que pueden derivar de lo anterior.

El tipo de socialización formativa y organizacional ${ }^{4}$ que ha recibido el profesorado puede relacionarse con el nivel de consolidación de la profesión académica. La presente investigación sobre la socialización del personal académico se realizó a nivel nacional, atendiendo sobre todo a la socialización formativa. Este trabajo tiene que ver con la manera de ser académico en México; indaga cómo el académico se ha ido socializando en las normas, valores y pautas de conducta que son propias de la profesión académica. Esta socialización inicia desde que el actual profesor recibió su preparación académica, especialmente durante el posgrado (maestría y doctorado). Los estudiosos de la socialización del profesorado ${ }^{5}$ denominan a esta etapa de formación académica, como la etapa de socialización anticipatoria. Durante esta fase los potenciales académicos entran en contacto, como estudiantes, con el "ethos" de la academia previamente a su incorporación en alguna institución de educación superior (IES) ${ }^{6}$ En el caso de México, a esta

2 B. Clark, The Academic Life: Small worlds, different worlds, Princeton, N.J., Carnegie Foundation for the Advancement of Teaching, 1987; T. Becher, Tribus $y$ territories académicos, Barcelona, Editorial Gedisa, 2001.

3 M. Gil Antón, op. cit.

${ }^{4}$ W. G. Tierney y E. M. Bensimon, Promotion Tenure. Community and Socialization in Academie, New York, N.Y., State University of New York Press, SUNY Series, 1996.

5 W. G. Tierney y R. A. Rhoads, Enhancing Promotion, Tenure, and beyond: Faculty Socialization as a Cultural Process. ASHE-ERIC Higher Education Report No. 6. Washington, D. C., The George Washington University, 1993.

- A. E. Austin, "Preparing the next generation of faculty. Graduate school as socialization to the academic career, en The Journal of Higher Education, 73, (1), pp. 94-122, 2002 
etapa se le reconoce más como de socialización formativa, ya que la preparación académica ocurre en ocasiones de manera simultánea a la trayectoria como profesor, no necesariamente antes ${ }^{7}$.

Este estudio se inscribe en la tradición de investigación sobre académicos iniciada en el país por Manuel Gil Antón y un equipo de colaboradores ${ }^{8}$, a finales de la década de 1980 en la Universidad Autónoma Metropolitana-Azcapotzalco. Sus resultados permiten identificar aspectos relevantes que pueden recomendarse para su atención en el conjunto de políticas y programas federales e institucionales orientados al desarrollo de la profesión académica en México.

\section{Metodología}

El estudio incluyó dos tipos de acercamientos. El primero, de corte más cuantitativo, se orientó a conocer el impacto de variables clave como el tipo de institución de formación y de adscripción, la disciplina de formación y de adscripción y el periodo de formación y de incorporación a la vida académica en la socialización de los académicos mexicanos, así como otras variables de tipo demográfico y de condiciones de trabajo. Lo anterior se realizó a partir de una base de datos construida como resultado del Estudio Nacional sobre el Impacto de las Políticas Públicas y el Cambio Institucional en la consolidación de la profesión académica en México; dicho estudio fue conducido por Rocío Grediaga y apoyado por el CONACyT ${ }^{9}$. En el estudio participaron 65 IES y respondieron el cuestionario 3,861 profesores de todo el país.

El segundo acercamiento fue de tipo más cualitativo, con la finalidad de profundizar en el conocimiento de la socialización del personal

7 R. Grediaga, Profesión académica, disciplinas y organizaciones, México, ANUIES, 2000; L. Villa Lever, "El mercado académico: la incorporación, la definitividad y las promociones, pasos para una misma trayectoria de formación, en Revista Mexicana de Investigación Educativa, VI, (1 1), pp. 63-78, 2001.

8 Manuel Gil Antón et al., op. cit., 1994.

9 R. Grediaga, R. Rodríguez y L. Padilla, Políticas públicas y cambios en la profesión académica en México en la última década, México, ANUIES, 2004.

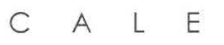

D

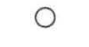

S

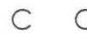

1

O


académico en México. Para ello se realizaron 34 entrevistas a profesores pertenecientes a 8 IES. Las IES se seleccionaron tratando de captar la diversidad institucional que caracteriza al país incluyendo instituciones públicas y privadas, y dentro de éstas a las confesionales; así mismo se consideró su mayor o menor orientación hacia la docencia o la investigación. En cuanto a los profesores, se procuró también un equilibrio atendiendo al nivel formativo, la disciplina, la dedicación a la institución, la edad y el género. Para el análisis de la información cualitativa se consideraron diez categorías: la primera, relacionada con el interés y motivación en la carrera académica; tres más relativas a las influencias significativas para la elección de la carrera académica durante la formación de licenciatura, de maestría y de doctorado; otras cuatro categorías relativas a la socialización organizacional a partir del proceso inicial de reclutamiento y hasta el ajuste e integración a la vida académica. Otra categoría describe la satisfacción con la carrera académica, y finalmente, la última agrupa las sugerencias para mejorar el desarrollo del personal académico.

Para el análisis de la información se consideraron centrales tres variables, el tipo de IES en que laboraban los profesores, el tipo de disciplina en que se agrupan y el periodo de inicio en la vida académica.

\section{REsultados y CoNCLUSIONES}

\section{Hallazgos demográficos}

En primer término se presentan los resultados que se destacan en el aspecto demográfico. En relación con el género, se observó que globalmente poco más de tres quintas partes (63\%) de los profesores en la muestra fueron hombres. Si se atiende sólo a los profesores que iniciaron su vida académica antes de 1970, se tiene que los hombres representaban el 78\%; mientras que este porcentaje disminuye a $56 \%$, si se atiende solo al profesorado que inició su carrera entre 1997 y 2001, es decir, se identifica una tendencia progresiva hacia el equilibrio de género en términos del número de plazas ocupadas por los académicos en las IES. En cuanto a la disciplina se observó que el área de las ingenierías concentró la menor proporción de mujeres, 
que fue de $19.8 \%$, mientras que las áreas de educación, comunicación, administración y negocios, etc. concentraron la mayor proporción de ellas (43\%). Como resultado de las entrevistas, se apreció en opinión de los profesores que las mayores diferencias en cuanto al género se perciben en la ocupación de cargos administrativos de alto nivel, que son ocupados en su mayoría por hombres, lo cual puede constituir un tema para futuras investigaciones.

En cuanto a la edad, se estableció una edad promedio de 44 años de los académicos en México, lo cual añade 3 años a la edad promedio reportada por Gil Antón y otros ${ }^{10}$ hace aproximadamente una década. De acuerdo con Altbach ${ }^{11}$ los académicos mexicanos se encuentran entre los más jóvenes comparados con otros países. La media de edad de los hombres (45.5 años) fue mayor que la de las mujeres (41.9 años), lo que se explica en parte por su incorporación más reciente a la academia. El 28\% del total de los académicos en la muestra fue mayor de 50 años y cerca del 40\% inició su carrera académica antes de 1985. Lo anterior puede contribuir a estimar la proporción de académicos que estarán retirándose de la academia en los próximos 20 años y plantea el problema tanto del retiro de los académicos actualmente en servicio, como el de las generaciones de reemplazo. Lo anterior representa también un campo necesario de análisis para futuras investigaciones. Finalmente, se observó que la edad de incorporación a la vida académica ha tendido a aumentar, pasando en promedio de 26 años antes de 1984 a 32 años entre 1997 y 2001. Es decir, el profesorado tiende a iniciar su vida académica a una edad mayor; en los últimos 20 años el promedio de edad de incorporación se ha incrementado en 6 años.

\section{Socialización formativa del personal académico en México}

A continuación se presentan los resultados más relevantes identificados en la socialización formativa de los académicos mexicanos. Se

\footnotetext{
10 Gil Antón et al., op. cit., 1994.

II Op. cit.
} 
confirma que la socialización durante la licenciatura continúa siendo la influencia más significativa en la decisión de iniciar una carrera académica $160 \%$ la inició inmediatamente después de ella, y $82 \%$ la inició con solo este grado o menos). Esta situación difiere sustancialmente de la formación que se requiere en IES de otros países para la incorporación a la academia, que es generalmente de nivel doctoral. Lo anterior es un primer indicador de la fragilidad en la formación disciplinaria del profesorado, a la que hacen mención otros estudios ${ }^{12}$. Además, la formación de licenciatura se orienta al desarrollo de habilidades para el ejercicio de la profesión que normalmente no contempla el desarrollo de competencias requeridas en el ámbito académico, como se evidencia en seguida.

Cabe resaltar que los resultados indican que cerca del $40 \%$ de los académicos señalan que la formación de licenciatura contribuyó de manera más enfática al desarrollo de sus habilidades profesionales, y en menor medida a sus habilidades docentes y de investigación. Aunque se encuentran diferencias por disciplina, ya que en las áreas puras-duras (física, química, biología, etc.) se tiende a valorar más la formación en investigación y en las aplicadas blandas (educación, comunicación, etc.), la formación en docencia; las diferencias por tipo de institución más importantes fueron las relativas a instituciones pequeñas no diversificadas, en donde las orientadas a pregrado y posgrado profesionalizante dan más importancia a las habilidades docentes (48\%) y las orientadas a investigación, correspondientemente, enfatizan las habilidades para la investigación (38\%).

Sin embargo, y dada la importancia del nivel de licenciatura para la socialización formativa del los futuros académicos, a través de las entrevistas no se identificó en ninguna IES la existencia de programas diseñados explícitamente para atraer y formar a los estudiantes talentosos y motivados, por el contrario, la socialización se dio mas bien de forma individual, informal y no secuencial ${ }^{13}$ y en gran medida el inicio

\footnotetext{
12 Gil Antón, Op. cit. 1994.

13 Tierney y Rhoads, Op. cit.
} 
de la vida académica se dio más como una decisión no planeada; esta resulta ser más el producto de circunstancia aleatorias vinculadas con la situación del mercado laboral de las profesiones específicas. La preocupación por formar adecuadamente a los futuros académicos se ha sentido en otros países; por ejemplo, en los Estados Unidos se inició en 1993 un programa de alcance nacional denominado Preparing Future Faculty, orientado a los estudiantes de doctorado y promovido por el Council of Graduate Schools y la Association of American Colleges and Univerisites (www.preparing-faculty.org).

Las entrevistas reflejaron que en ocasiones el interés de los estudiantes por seguirse formando a nivel de posgrado y el apoyo que tuvieron de algún profesor para ello fue una de las influencias más significativas para crear el vínculo con el inicio de la vida académica. Varios académicos entrevistados indicaron haber sido adjuntos o asistentes de algún profesor cuando estudiaban su licenciatura y que esa experiencia los acercó a la vida académica; aunque también hicieron notar que esas figuras tienden a desaparecer en la actualidad, sobre todo debido a la falta de recursos económicos. Los profesores entrevistados señalaron que en su decisión de iniciar una carrera académica influyó el que se percibieran a sí mismos como "buenos estudiantes" en un "entorno educativo desafiante" y que además sus compañeros los buscaban para que les ayudaran a estudiar. De alguna manera, en la interacción con los compañeros encontraron que tenían facilidad para enseñar y así ayudar a los demás.

De acuerdo con resultados de este estudio, el $82 \%$ de la planta académica en la muestra inició su trayectoria con sólo el título de licenciatura (56\%) o menos (26\%); mientras que sólo un $8 \%$ contaba con estudios de maestría y un $2 \%$ de doctorado. Estos resultados confirman lo encontrado por estudios previos ${ }^{14}$. Esta situación tiende a cambiar con el tiempo, ya que mientras antes de 1985, entre una tercera y dos quintas partes de los profesores que se incorporaron fueron contratados sin contar con licenciatura, entre 1996 y 2001 dicha

14 Gil Antón et al., 1994

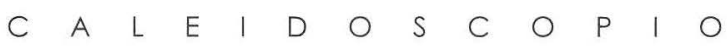


proporción apenas alcanzó la décima parte. Sin embargo, se observó otro fenómeno que puede denominarse como movilidad escolar y que se expresó en el hecho de que cerca de un 57\% del profesorado en la muestra agregó entre 1 y 4 niveles o grados académicos a su formación inicial durante su trayectoria como académicos. Por esta razón, a esta etapa socializadora se denomina más apropiadamente formativa que anticipatoria, como ya se mencionó. En el año 2001, en comparación con el año de incorporación a la vida académica, la proporción de profesores con maestría se elevó de $8.3 \%$ a $31.1 \%$, y la de doctorado de $1.9 \%$ a 10.5\%. La formación inicial de los académicos y la alcanzada en 2001, se presenta en el Cuadro 1.

Cuadro 1. Grado académico de inicio y alcanzado en 2001 por periodo de ingreso a la vida académica. (frecuencias y porcentajes por periodo)

\begin{tabular}{|c|c|c|c|c|c|c|c|c|}
\hline \multirow{2}{*}{$\begin{array}{l}\text { Periodo de } \\
\text { inicio en la } \\
\text { vida acadé- } \\
\text { mica }\end{array}$} & \multicolumn{3}{|c|}{$\begin{array}{l}\text { Grado máximo } \\
\text { al inicio }\end{array}$} & \multicolumn{3}{|c|}{$\begin{array}{l}\text { Grado máximo en } \\
2001\end{array}$} & \multirow{2}{*}{$\begin{array}{c}\text { Total } \\
\text { N } \\
\%\end{array}$} & \multirow{2}{*}{$\begin{array}{c}\begin{array}{c}\text { No } \\
\text { movilli- } \\
\text { dad }\end{array} \\
\mathrm{N} \\
\%\end{array}$} \\
\hline & $\begin{array}{l}\text { Li- } \\
\text { cen- } \\
\text { cia- } \\
\text { tura }\end{array}$ & $\begin{array}{l}\text { Ma- } \\
\text { es- } \\
\text { tría }\end{array}$ & $\begin{array}{l}\text { Doc- } \\
\text { to } \\
\text { rado }\end{array}$ & $\begin{array}{l}\text { Licen- } \\
\text { ciatu- } \\
\text { ra }\end{array}$ & $\begin{array}{l}\text { Ma- } \\
\text { es- } \\
\text { tría }\end{array}$ & $\begin{array}{l}\text { Doc- } \\
\text { to- } \\
\text { rado }\end{array}$ & & \\
\hline $\begin{array}{l}1970 \text { o } \\
\text { anterior } \\
\text { Expansión } \\
\text { Moderada }\end{array}$ & $\begin{array}{r}115 \\
48.3\end{array}$ & $\begin{array}{l}21 \\
8.8\end{array}$ & $\begin{array}{c}1 \\
.4 \%\end{array}$ & $\begin{array}{c}75 \\
31.5\end{array}$ & $\begin{array}{c}75 \\
31.5\end{array}$ & $\begin{array}{c}44 \\
18.5\end{array}$ & $\begin{array}{l}238 \\
6.2 \%\end{array}$ & $\begin{array}{c}66 \\
27.7 \%\end{array}$ \\
\hline $\begin{array}{l}1971 \text { a } 1984 \\
\text { Expansión } \\
\text { Acelerada }\end{array}$ & $\begin{array}{l}631 \\
47.8\end{array}$ & $\begin{array}{l}76 \\
5.8\end{array}$ & $\begin{array}{l}10 \\
.8 \%\end{array}$ & $\begin{array}{c}445 \\
33.7 \%\end{array}$ & $\begin{array}{l}426 \\
32.3\end{array}$ & $\begin{array}{l}205 \\
15.5\end{array}$ & $\begin{array}{l}1320 \\
34.2\end{array}$ & $\begin{array}{c}357 \\
27.0 \%\end{array}$ \\
\hline $\begin{array}{l}1985 \text { a } 1990 \\
\text { Desacelera- } \\
\text { ción }\end{array}$ & $\begin{array}{r}425 \\
56.1\end{array}$ & $\begin{array}{l}54 \\
7.1 \\
\end{array}$ & $\begin{array}{c}11 \\
1.5 \%\end{array}$ & $\begin{array}{c}284 \\
37.5 \%\end{array}$ & $\begin{array}{l}286 \\
37.7\end{array}$ & $\begin{array}{c}67 \\
8.8 \%\end{array}$ & $\begin{array}{l}758 \\
19.6\end{array}$ & $\begin{array}{c}278 \\
36.7 \%\end{array}$ \\
\hline $\begin{array}{l}1991 \text { a } 1996 \\
\text { Evaluación }\end{array}$ & $\begin{array}{l}461 \\
62.0\end{array}$ & $\begin{array}{l}74 \\
9.9 \\
\end{array}$ & $\begin{array}{c}20 \\
2.7 \%\end{array}$ & $\begin{array}{c}332 \\
44.6 \%\end{array}$ & $\begin{array}{l}249 \\
33.5\end{array}$ & $\begin{array}{c}51 \\
6.9 \% \\
\end{array}$ & $\begin{array}{l}744 \\
19.3\end{array}$ & $\begin{array}{c}380 \\
51.1 \%\end{array}$ \\
\hline $\begin{array}{l}1997 \text { a } 2001 \\
\text { PROMEP }\end{array}$ & $\begin{array}{l}521 \\
65.0\end{array}$ & $\begin{array}{c}95 \\
11.9\end{array}$ & $\begin{array}{c}30 \\
3.7 \%\end{array}$ & $\begin{array}{c}459 \\
57.3 \%\end{array}$ & $\begin{array}{l}165 \\
20.6\end{array}$ & $\begin{array}{c}39 \\
4.9 \%\end{array}$ & $\begin{array}{l}801 \\
20.7\end{array}$ & $\begin{array}{c}578 \\
72.2 \%\end{array}$ \\
\hline Total & $\begin{array}{l}2153 \\
55.8\end{array}$ & $\begin{array}{l}320 \\
8.30\end{array}$ & $\begin{array}{c}72 \\
1.9 \%\end{array}$ & $\begin{array}{r}1595 \\
41.3 \%\end{array}$ & $\begin{array}{l}1201 \\
31.1\end{array}$ & $\begin{array}{l}406 \\
10.5\end{array}$ & $\begin{array}{l}3861 \\
100.0\end{array}$ & $\begin{array}{c}1659 \\
43.0 \%\end{array}$ \\
\hline
\end{tabular}

Además, la movilidad escolar se aceleró a partir de 1990, ya que cerca del $50 \%$ de los profesores que iniciaron estudios de posgrado (maestría y doctorado) lo hicieron entre 1990 y 2001 . Lo anterior indica 
el potencial impacto que ciertas políticas internacionales, federales e institucionales han podido tener en la formación de la planta académica, específicamente las del Programa de Superación Académica SUPERA, las del Programa de Mejoramiento del Profesorado PROMEP y las de agencias acreditadoras a las que recurren principalmente IES particulares, como la Southern Association of Colleges and Schools (SACS) de los Estados Unidos y los indicadores de la propia Federación de Instituciones Mexicanas Particulares de Educación Superior (FIMPES), ya que este fenómeno no es exclusivo de IES públicas.

A pesar del importante avance en la formación del personal académico, todavía la proporción de éste con solo el nivel de licenciatura es muy grande, lo cual puede relacionarse con la gran proporción de profesores que se contratan solo por asignatura, en donde las políticas de formación de académicos han incidido mucho menos. Además, se observó que las IES siguen contratando académicos con solo el grado de licenciatura, ya que por ejemplo, el 65\% de los profesores que iniciaron su vida académica entre 1997 y 2001 contaban solo con el grado de licenciatura. De seguir esta tendencia la socialización formativa difícilmente será anticipatorio y las IES y el gobierno federal requerirán destinar una importante cantidad de recursos a procurar elevar el grado académico del profesorado mexicano.

\section{Condiciones de la formación de posgrado}

En relación con la formación de maestría se encontró que solo el 8\% de los académicos había iniciado su vida académica con el grado de maestría, mientras que el $31 \%$ lo había obtenido en el momento de la entrevista; este porcentaje incluye entonces sólo a los académicos con el grado alcanzado. No obstante, se identificó que el 57\% de los académicos (2194 profesores) había, además de haber alcanzado el grado, iniciado estudios de maestría y los tenía en curso o bien estaba trabajando en su tesis. El análisis de las condiciones de formación se hace sobre esta última proporción. Para el análisis de las condiciones de estudio se considera, al igual que para la maestría, no solo los profesores que habían alcanzado el grado sino lo que habían iniciado o estaban en proceso de graduación de un programa de posgrado, en este caso la proporción se eleva del $2 \%$ al 19\% (733 profesores).

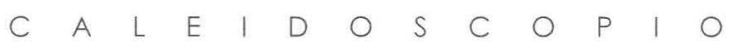


No obstante que se presentaron importantes niveles de movilidad escolar, las condiciones de socialización formativa, es decir, aquéllas que describen cómo se llevaron a cabo los estudios de posgrado (como la dedicación completa al estudio, si los académicos contaron con beca, si realizaron tesis, entre otras), no fueron en muchos casos las deseables. Lo anterior puede observase en el Cuadro 2, en donde se aprecia que sólo cerca de la mitad de los que iniciaron un programa de maestría lo hicieron como estudiantes de tiempo completo y con beca, además el 70\% reporta haber trabajado durante sus estudios. En rela ción con los estudios del doctorado, las condiciones fueron un poco más favorables ya que cerca de dos terceras partes de los académicos que iniciaron estos estudios lo hicieron de tiempo completo y con beca y sólo el 65\% reporta haber trabajado durante los estudios. Aún así, se observó que proporciones importantes de académicos (cerca del $50 \%$ para la maestría y del 35\% para el doctorado) no contaron con condiciones favorables para el estudio como el poderse dedicar de tiempo completo a éste o tener una beca como apoyo.

Cuadro 2. Condiciones de Estudio de la Maestría y del Doctorado por principales tipos de IES (Porcentaje de académicos por renglón)

\begin{tabular}{|c|c|c|c|c|}
\hline \multirow[t]{2}{*}{$\begin{array}{l}\text { Tipo de IES en donde } \\
\text { Se obtuvo el grado de } \\
\text { maestría }\end{array}$} & $\begin{array}{c}\text { Estudió } \\
\text { Tiempo } \\
\text { completo }\end{array}$ & $\begin{array}{c}\text { Contó } \\
\text { con } \\
\text { beca }\end{array}$ & $\begin{array}{c}\text { Trabajó } \\
\text { Durante } \\
\text { estudios }\end{array}$ & $\begin{array}{c}\text { Total } \\
\mathrm{N} / \% \text { por } \\
\text { Columna }\end{array}$ \\
\hline & $\begin{array}{l}\text { Maest./ } \\
\text { Doct. }\end{array}$ & $\begin{array}{l}\text { Maest./ } \\
\text { Doct. }\end{array}$ & $\begin{array}{l}\text { Maest./ } \\
\text { Doct. }\end{array}$ & $\begin{array}{l}\text { Maest./ } \\
\text { Doct. }\end{array}$ \\
\hline $\begin{array}{l}\text { Instituciones Grandes } \\
\text { diversificadas }\end{array}$ & $\begin{array}{l}38.2 \\
50.0 \\
\end{array}$ & $\begin{array}{l}52.9 \\
62.2 \\
\end{array}$ & $\begin{array}{l}81.3 \\
78.4 \\
\end{array}$ & $\begin{array}{c}694,31.6 \% \\
74,10.1 \%\end{array}$ \\
\hline $\begin{array}{l}\text { Instituciones Grandes } \\
\text { Diversificadas de la Zona Metro- } \\
\text { politana de Cd. de México. }\end{array}$ & $\begin{array}{l}58.2 \\
53.3\end{array}$ & $\begin{array}{l}52.0 \\
52.3\end{array}$ & $\begin{array}{l}69.9 \\
79.1\end{array}$ & $\begin{array}{ll}691, & 31.5 \% \\
321, & 43.8 \%\end{array}$ \\
\hline $\begin{array}{l}\text { Instituciones pequeñas centra- } \\
\text { das en investigación y posgrado } \\
\text { (Padrón de Excel.) }\end{array}$ & $\begin{array}{l}82.4 \\
86.7\end{array}$ & $\begin{array}{l}75.9 \\
88.7\end{array}$ & $\begin{array}{l}41.2 \\
36.5\end{array}$ & $\begin{array}{l}187,8.5 \% \\
203,27.7\end{array}$ \\
\hline IES extranjeras & $\begin{array}{l}49.1 \\
53.9\end{array}$ & $\begin{array}{l}53.5 \\
66.7\end{array}$ & $\begin{array}{l}66.1 \\
70.6\end{array}$ & $\begin{array}{ll}495, & 22.6 \% \\
102 . & 13.9 \%\end{array}$ \\
\hline Total: N con Maest./Doct.* & $\begin{array}{l}50.9 \\
62.5\end{array}$ & $\begin{array}{l}54.7 \\
65.3\end{array}$ & $\begin{array}{l}70.3 \\
65.1\end{array}$ & $\begin{array}{ll}2194, & 57 \% \\
733 & 19 \%\end{array}$ \\
\hline
\end{tabular}

*El total de la suma de los académicos no coincide con la suma de los académicos en las categorías de IES porque el cuadro no desglosa todos lo tipos considerados. 
El mismo Cuadro 2, presenta estas condiciones de acuerdo con los cuatro tipos de IES que concentraron al mayor número de los académicos durante sus estudios. Es evidente que las mejores condiciones formativas (muy por encima de los porcentajes globales) las tuvieron los académicos que estudiaron en IES centradas en investigación y posgrado, sin embargo, sólo el $8.5 \%$ de los que realizaron maestría lo hicieron en este tipo de IES, mientras que esta proporción fue más significativa en los estudios de doctorado (27.7\%). Las IES grandes diversificadas, tanto de la zona metropolitana de la Ciudad de México (31.5\%) como las ubicadas en el resto del país (31.6\%) concentraron las mayores proporciones de académicos que iniciaron un programa de maestría (cerca de dos terceras partes). Los académicos que estudiaron en el extranjero representan cerca de la quinta parte en el nivel de maestría y el $14 \%$ en el nivel de doctorado. En este último nivel, son claramente las IES grandes diversificadas de la zona metropolitana de la Ciudad de México quienes concentran al 44\% de los académicos, seguidos de las IES orientadas a investigación y posgrado.

Es importante señalar también la heterogeneidad de la calidad y exigencia de los programas de posgrado en los que los académicos se han estado formando. Las entrevistas reflejan que el interés en el posgrado se manifiesta como un reflejo de las exigencias actuales de las IES, tanto públicas como privadas, ya que ahora se les requiere mínimo maestría para poder impartir clase, aunque sean profesores por asignatura, además de que los profesores que tienen maestría tienen ventajas sobre los que no la tienen. Esta situación lleva a preguntarse si el tener el nivel de maestría y de doctorado refleja realmente el dominio de determinados conocimientos, habilidades, y actitudes que son propios de estos niveles formativos o el profesor que logra el grado está más interesado en cumplir con un requisito que le impone la institución de educación superior donde labora.

\section{¿ENDOGAMIA ACADÉMICA?}

Al considerar la relación entre la región geográfica de nacimiento de los académicos en el estudio y la del trabajo actual, se desprende la existencia de una baja movilidad geográfica, ya que cerca del 75\% del profesorado de cada región tiende a permanecer en ella. Este patrón 
de baja movilidad geográfica puede estar relacionado también con la tendencia de las IES a contratar a los propios egresados y que se refiere en la literatura como "endogamia académica" (academic inbreeding), y con el patrón de baja movilidad interinstitucional que ha sido destacado en diversos estudios sobre los académicos del país ${ }^{15}$. Estos aspectos, a su vez podrían enmarcarse en patrones culturales más amplios asociados con los lazos familiares, tema que constituye una importante veta para futuros estudios.

La tendencia de las IES a contratar a los propios egresados es difícil de observar ya que depende en parte de si ésta se considera en función del grado máximo obtenido o de cuál de ellos, así como de si esta tendencia se presenta inmediatamente egresando o después de un lapso de tiempo. En este caso, esta tendencia se valoró a partir del grado máximo de estudios y se contrastó tanto con la IES en que el académico laboró por primera ocasión como en la IES en que trabaja actualmente. Grediaga y otros ${ }^{16}$ encontraron que esta tendencia ha disminuido, pues menos de la mitad de los contratados a partir de 1996 (47.7\%) fueron contratados por la misma institución en que habían obtenido el grado máximo alcanzado al momento de iniciar sus trayectorias académicas, cuando en dos periodos previos esta proporción era superior a la mitad de los contratados $153.5 \%$ en el segundo y $54.9 \%$ en el tercero). Cabe señalar que cerca del $76 \%$ de los profesores en el estudio estaba laborando en la misma IES en que fue contratado por primera ocasión (baja movilidad interinstitucional).

Las entrevistas realizadas permitieron identificar que en ocasiones los académicos regresan a trabajar a las instituciones en que fueron formados durante la preparatoria, no sólo durante la licenciatura, especialmente cuando estas instituciones se distinguen por una formación con una explícita filosofía educativa. La tendencia de las IES a

15 Gil, et al. op. cit. 1994; Grediaga, op.cit.; J. F. Galaz, Jo6 satisfaction of Mexican faculty in a public state university: Institutional reality through the lens of the professoriate, Dissertation submitted to the faculty of Claremont University, Claremont, CA, Disertation, 2002; Grediaga, Rodríguez y Padilla, op.cit.

16 Grediaga et al. op. cit., 2004. 
contratar a sus propios egresados por un lado facilita la integración de los nuevos académicos y la continuidad de la cultura organizacional, sin embargo también se puede propiciar sectarismo, estancamiento y estrechez de miras. Sin duda, este aspecto del profesorado mexicano requiere de estudios más detallados.

Finalmente, en relación con las actividades de los académicos, la enseñanza continuó siendo la principal actividad desarrollada por ellos ${ }^{17}$. La productividad del profesorado, aún considerando productos derivados de docencia, fue baja, así como la participación en asociaciones científicas a nivel tanto nacional como internacional. La tensión entre la docencia y la investigación se reflejó también en las entrevistas, en donde se percibe que la situación de los investigadores es más valorada y bien remunerada por las IES. La realización de investigación se presenta en general para aquellos profesores que son de tiempo completo y con nivel formativo alto, lo cual no es el perfil del académico que prevalece en las IES mexicanas. Los profesores por asignatura tienen menos oportunidades de participación incluso también en el proceso de toma de decisiones que le afectan.

La situación descrita hasta ahora puede variar si se consideran los ejes centrales de diferenciación del profesorado ${ }^{18}$ es decir, el área disciplinar en que fueron formados los académicos y el tipo de IES en que se labora. Los académicos adscritos a IES diversificadas, principalmente de la zona metropolitana y de áreas puras duras presentaron las mejores condiciones de socialización tanto formativa como organizacional.

\section{RecomendaCiones FinAlES}

Dado que la socialización formativa para el profesorado mexicano sigue siendo en gran medida la licenciatura sería conveniente que las IES desarrollaran programas orientados a identificar y apoyar jóvenes

17 Altbach, op . cit.

18 Clark, op cit.

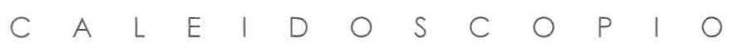


talentosos que pudieran interesarse en la carrera académica, desde el nivel de licenciatura pero también en el posgrado.

Las diferencias en la movilidad formativa de los profesores hacen pensar que el dinamismo y el liderazgo propio de cada área académica y disciplinas, así como de cada IES, es un factor que puede repercutir en el nivel formativo del profesorado. Por tanto es importante que las autoridades académicas de cada área e institución sean concientes de la importancia de la socialización formativa de los académicos bajo su responsabilidad, y de cómo impulsarla de manera eficaz.

Las políticas de contratación de académicos deben de considerar el grado máximo del aspirante, pero también el tipo de posgrado y el tipo de institución en donde se formó. Se considera que si bien existe un impacto de las políticas de PROMEP (en el sentido de incrementar el nivel formativo del profesorado para otorgar una plaza), todavía existe una gran proporción de académicos que requerirán de formación en programas estructurados de posgrado una vez iniciada su vida académica. Lo anterior requerirá una gran proporción de recursos para que estos incrementen su escolaridad una vez iniciada su vida académica.

Las políticas para mejorar el nivel formativo de los profesores tienen que considerar las importantes diferencias que se presentan por disciplina de adscripción. También es conveniente considerar las exigencias de formación que tiene el personal académico contratado por asignatura, que en general presenta condiciones de formación más desfavorables.

Hasta ahora, el impacto de las políticas de formación del personal académico impulsadas a nivel federal desde la década de los noventa se manifiesta con claridad en la alta proporción de los académicos (cerca del 50\%) que inició sus estudios de maestría y doctorado a partir de entonces. Sin embargo, las condiciones de formación no han sido las mejores por lo que se puede cuestionar la eficacia de esta formación y la manera como esta impacta realmente la calidad académica del profesorado y en última instancia de la propia institución. 